\section{La pourpre de Méditerranée, une source inattendue d'inhibiteurs de GSK-3}

Laurent Meijer
> La légende raconte qu'Héraclès, marchant sur une plage des côtes du Liban, observa la gueule colorée de violet d'un chien ayant croqué un mollusque. La découverte de la «pourpre royale» qui allait faire la réputation et la fortune des Phéniciens était faite. Pendant de nombreux siècles, les Phéniciens allaient en effet récolter Murex brandaris et Hexaplex trunculus jusqu'à entraîner leur extinction presque complète (on trouve en divers endroits de la côte du Levant, des collines de plusieurs mètres d'épaisseur faites de coquilles brisées de ces deux gastéropodes !) afin d'en extraire la précieuse «pourpre de Méditerranée» dont la couleur unique était un luxe réservé aux plus hauts dignitaires [1]. II fallut attendre 1909 pour que P. Friedlander isole 1,4 g du colorant à partir de 12000 Murex, et qu'il identifie la molécule colorée principale comme le 6,6'-dibromoindigo [2]. L'indigo était déjà bien connu, comme un bis-indole naturel obtenu par macération de diverses plantes et utilisé comme colorant bleu par l'homme depuis des millénaires [3]. Tout comme l'indigo extrait de plantes est contaminé par l'indirubine, un autre bis-indole isomère de l'indigo, la pourpre tirée des Mollusques s'est révélée contenir, outre le 6,6'-dibromoindigo dominant, différentes indirubines bromées (Figure 1).

Dans les années 1960, l'Académie de Médecine Chinoise étudiait le Danggui Longhui Wan, un mélange de plantes efficace contre diverses affections dont la leucémie myéloïde chronique, afin d'en purifier le principe actif. Par élimination sépa- rée de chacun des 11 ingrédients qui constituent cette préparation, suivie de tests directs chez l'homme, elle démontra clairement qu'un seul ingrédient, le Xing Dai, portait l'activité thérapeutique. Cet ingrédient était justement une poudre bleu sombre, extraite de plantes produisant de l'indigo ! II ne fallut guère de temps aux chercheurs pour se rendre compte que l'indigo était inactif mais qu'un contaminant mineur, l'indirubine, était responsable de l'activité anti-leucémique. L'histoire aurait pu en rester là car l'indirubine est un composé vraiment peu soluble et, malgré les efforts des chimistes chinois, peu de dérivés présentèrent une pharmacologie plus favorable. De plus, le mécanisme d'action de l'indirubine était totalement inconnu, ce qui contribua encore davantage à réduire l'intérêt du monde occidental pour ce produit pourtant actif sur les leucémies.

En 1999, au cours de criblages de composés issus de la pharmacopée chinoise sur une cible enzymatique bien définie, nous avons découvert que l'indirubine et ses dérivés étaient des inhibiteurs de certaines kinases, dont l'activité dépendait des cyclines (CDK), et qui étaient impliquées directement dans le contrôle de la division cellulaire, ce qui pouvait expliquer en partie l'effet antiprolifératif des indirubines [4, 5]. La cocristallisation de diverses indirubines avec CDK2 [4], CDK2/cycline A [6] et une kinase de Plasmodium falciparum, PfPK5 [7], a montré que ces petites molécules s'insèrent dans la poche de fixation de I'ATP des kinases, bloquant ainsi directement leur activité cataly-
CNRS, Groupe cycle

cellulaire,

Station Biologique,

29682 Roscoff cedex, France.

meijer@sb-roscoff.fr tique. En 2001, nous avons observé que les indirubines sont également des inhibiteurs puissants de la glycogène synthase kinase-3 (GSK-3) [8], une kinase aux multiples implications, intervenant dans la voie de signalisation Wnt, la mort cellulaire, la formation des axes pendant le développement, la maladie d'Alzheimer, la voie de signalisation de I'insuline...

Dans notre recherche de nouvelles indirubines plus puissantes et plus sélectives, nous nous sommes tournés vers les Mollusques marins. À partir de $60 \mathrm{~kg}$ d'H. trunculus récoltés en Grèce, notre collaborateur L. Skaltsounis est parvenu à extraire suffisamment de pourpre pour en purifier les différents constituants [9]. En plus du 6,6'-dibromoindigo attendu, la pourpre se révéla contenir diverses indirubines plus ou moins bromées (Figure 1). Elles ont toutes été synthétisées et complémentées d'une petite modification (3'-oxime) qui améliore sensiblement leur solubilité et leur efficacité (Figure 1). De façon inattendue, la 6-bromo-indirubine-3'-oxime (BIO), s'est révélée particulièrement active et nettement plus sélective vis-à-vis de la GSK-3 [9, 10]. Une collaboration avec les groupes d'A. Musacchio et de L. Pearl a permis d'obtenir la cocristallisation, respectivement, de l'indirubine-3'-oxime avec CDK5 et de BIO avec GSK-3, confirmant l'interaction des indirubines avec la poche de fixation de l'ATP des kinases et permettant d'expliquer les raisons d'une sélectivité accrue vis-à-vis de GSK-3 [11]. De nouveaux dérivés de BIO sont 
maintenant disponibles, 7000 fois plus actifs sur GSK-3 que sur les CDK.

Plusieurs modèles cellulaires nous ont permis de vérifier que BIO (mais pas son dérivé inactif MeBIO) inhibe effectivement GSK-3 à l'intérieur des cellules. $\varepsilon n$ particulier, en collaboration avec A. Brivanlou et son équipe, nous avons démontré que BIO mime parfaitement les effets de l'activation de la voie Wnt dans l'embryon précoce de xénope [9].
Mais les indirubines devaient nous révéler d'autres surprises. En effet, il apparaît que BIO (mais pas MeBIO) est capable de maintenir les cellules souches embryonnaires souches (ES, embryonic stem cells) à l'état indifférencié, sans toutefois empêcher leur multiplication [12]. Un produit chimique aisément synthétisable semble donc capable de remplacer les cellules stromales murines nécessaires au maintien des cellules ES dans un état indifférencié. De plus le phénomène est parfaitement réversible, puisque les cellules ES se différencient dès que BIO est éliminé du milieu de culture. BIO pourrait donc constituer un outil formidable pour maintenir indéfiniment des cellules ES à l'état indifférencié et donc obtenir des lignées, offrant ainsi une solution au problème éthique posé par l'utilisation d'embryons humains comme source de
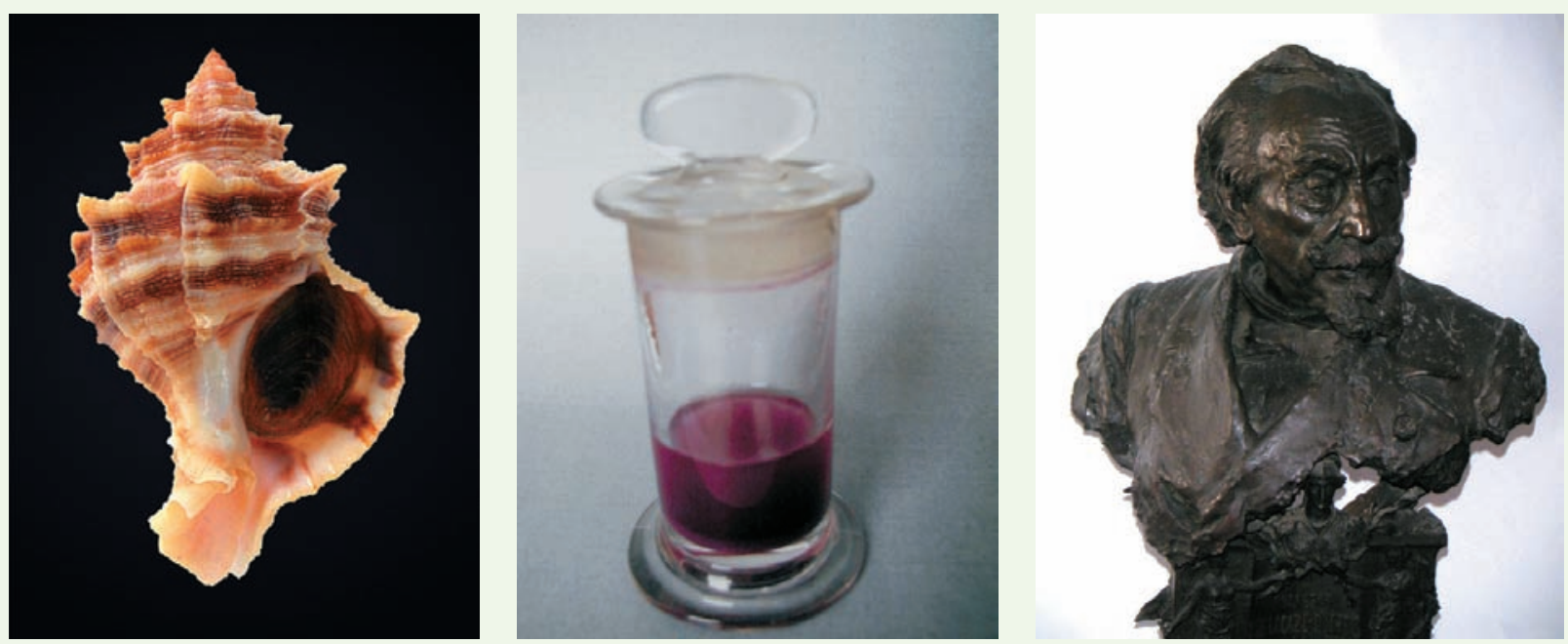<smiles>[Y]c1ccc2c(c1)N/C(=C1/Nc3cc([Y])ccc3C1=O)C2=O</smiles>

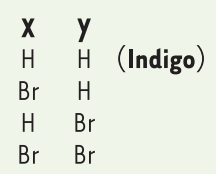<smiles>[Y]c1ccc2c(c1)[CH]C(=O)/C2=C1\C(=O)Nc2cc([Y])ccc21</smiles>

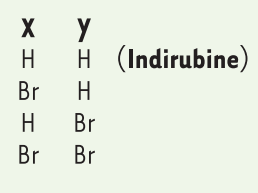<smiles>[Y]c1ccc2c(c1)N/C(=C1/C(=O)N([R])c3cc([Y])ccc31)C2=NO</smiles>

$\begin{array}{cccl}\mathbf{X} & \mathbf{y} & \mathbf{R} & \\ \mathrm{H} & \mathrm{H} & \mathrm{H} & (\mathbf{I 0}) \\ \mathrm{H} & \mathrm{Br} & \mathrm{H} & (\mathbf{B I O}) \\ \mathrm{H} & \mathrm{Br} & \mathrm{Me} & (\text { MeBIO })\end{array}$

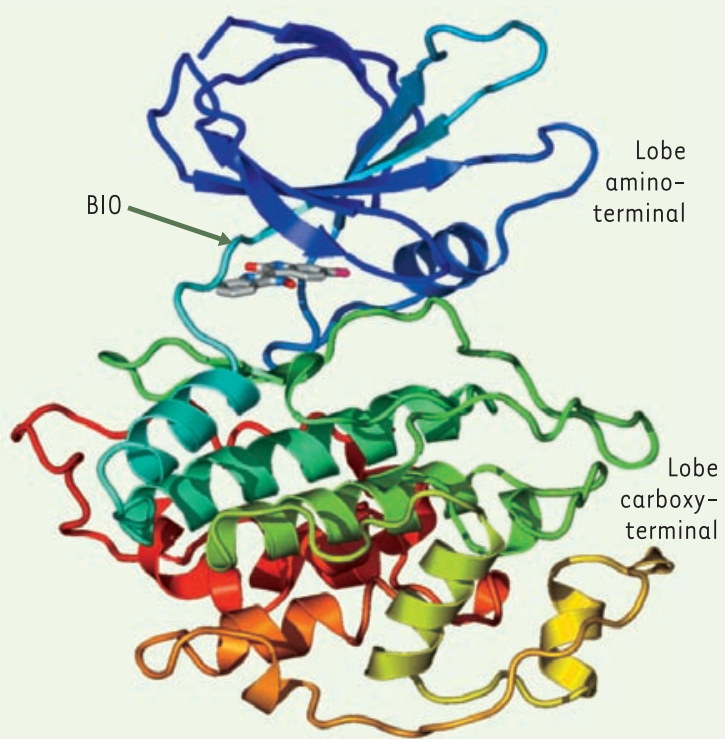

Figure 1. L'histoire de l'indirubine. Hexaplex trunculus, un mollusque gastéropode dont la glande hypobranchiale sécrète les précurseurs de la pourpre de Méditerranée, étudiée de longue date et notamment par Henri de Lacaze-Duthiers (photo), fondateur de la station biologique de Roscoff. La pourpre est constituée d'un mélange de bis-indoles (indigo et indirubine), plus ou moins bromés. Parmi les indirubines dérivées de ces substances naturelles, l'indirubine-3'-oxime (10), la 6-bromo-indirubine-3'-oxime (BI0) et son analogue inactif, la l-méthyl-6-bromo-indirubine-3'-oxime (MeBIO). La structure du co-cristal BIO/GSK-3ß révèle que l'inhibiteur occupe la poche de fixation de l'ATP (photographies, P. Monfils et S. Bach). 
cellules ES. La prolifération de ces cellules ES dans un milieu défini, n'ayant pas été exposé aux tissus et cellules d'autres mammifères, permet d'envisager leur utilisation chez l'homme sans risque de contamination par des virus apportés par les cellules stromales nourricières. La voie de la médecine régénérative semble plus ouverte que jamais! Lorsqu'en 1826, âgé de 23 ans, AntoineJérome Balard (qui donna son nom à une station de métro et à une place parisienne) découvrait dans l'eau de mer un nouvel élément, il proposa le nom de Murine à l'Académie, en raison de sa couleur pourpre et de son extraction à partir de saumure. L'Académie lui préféra le nom de Brome (du grec Bromos, mauvaise odeur) [13]. Celui qui allait devenir le directeur de thèse de Louis Pasteur pouvait-il imaginer que les molécules responsables de la couleur si caractéristique de la pourpre contenaient bien l'élément qu'il avait identifié ? Quelques décennies plus tard, Henri de Lacaze Duthiers, fondateur de la Station Biologique de Roscoff, étudiait en détail la production de pourpre par les mollusques Muricidés [14]. Pouvait-il deviner que 150 ans plus tard, la pourpre de Tyr intéresserait encore les chercheurs et qu'elle laisserait entrevoir des applications diverses? Les indirubines ont certainement encore beaucoup de secrets à nous révéler!

À suivre... $\diamond$

Tyrian purple indirubins:

a source of GSK-3 inhibitors

\section{RÉFÉRENCES}

1. Cooksey CJ. Tyrian purple: 6,6'-dibromoindigo and related compounds. Molecules 2001; 6: 736-69.

2. Friedlander P. Uber den Farstoff des antikeen Purpurs aus Murex brandaris. 1909 ; 765-70.

3. Balfour-Paul J. Indigo. British Museum Press. 1998 : $264 \mathrm{p}$.

4. Hoessel R, Leclerc $S$, Endicott J, et al. Indirubin, the active constituent of a Chinese antileukaemia medicine, inhibits cyclin-dependent kinases. Nat Cell Biol 1999; 1: 60-7.

5. Damiens $\varepsilon$, Baratte B, Marie D, et al. Anti-mitotic properties of indirubin-3'-monoxime, a CDK/GSK-3 inhibitor: induction of endoreplication following prophase arrest. Oncogene 2001; 20: 3786-97.

6. Davies TG, Tunnah P, Meijer L, et al. Inhibitor binding to active and inactive CDK2. The crystal structure of a CDK2-cyclin A/indirubin-5-sulphonate. Structure 2001; 9: 389-97.

7. Holton S, Merckx A, Burgess D, et al. Structures of P. falciparum PfPK5 test the CDK regulation paradigm and suggest mechanisms of small molecule inhibition. Structure 2003; 11: 1329-37.

8. Leclerc $S$, Garnier M, Hoessel R, et al. Indirubins inhibit glycogen synthase kinase- $3 \beta$ and CDK5/p25, two kinases involved in abnormal tau phosphorylation in Alzheimer's disease - a property common to most CDK inhibitors? J Biol Chem 2001; 276: 251-60.

9. Meijer L, Skaltsounis AL, Magiatis P, et al. GSK-3 selective inhibitors derived from Tyrian purple indirubins. Chem Biol 2003; 10: 1255-66.

10. Polychronopoulos $P$, Magiatis $P$, Skaltsounis $L$, et al. Structural basis for the synthesis of indirubins as potent and selective inhibitors of glycogen synthase kinase- 3 and cyclin-dependent kinases. J Med Chem 2004; 47: 935-46.

11. Fischer PM. CDK versus GSK-3 inhibition: a purple haze no longer? Chem Biol 2003; 10: 1144-6.

12. Sato N, Meijer L, Skaltsounis L, et al. Maintenance of pluripotency in human and mouse embryonic stem cells through activation of Wnt signaling by a pharmacological GSK-3 specific inhibitor. Nat Med 2004; 10: 55-63.

13. Dolique R. Balard, sa vie, son œuvre à Montpellier de 1802 à 1840. Rev Hist Pharm 1977; 232: 13-27.

14. Lacaze-Duthiers H. Mémoire sur la Pourpre. An Sci Nat Zool 1859; 12: 5-84.

\section{NOUVELLE}

\section{Des cellules souches pour corriger les surdités neurosensorielles?}

Azel Zine

> L'audition et l'équilibre dépendent de l'intégrité de l'oreille interne, composée de deux parties: la cochlée, responsable de l'audition, et le vestibule, responsable de l'équilibre (Figure I). L'épithélium neurosensoriel de la cochlée des mammifères, ou organe de Corti, comprend deux types de cellules: les cellules sensorielles, ou cellules ciliées, internes ( $\mathrm{CCl}$ ) et externes (CCE) (Figure 1), responsables de la transduction auditive, et les cellules de soutien.

Dans l'oreille interne des mammifères, le nombre des cellules sensorielles est fixé définitivement après la phase de différenciation cellulaire. Chez l'homme, on dénombre ainsi en moyenne $3500 \mathrm{CCl}$ et $12000 \mathrm{CC} \varepsilon$ dans une cellules ciliées $(\rightarrow)$. cochlée différenciée, dès le
Inserm U.583, Université Montpellier I, 80 , rue Augustin Fliche, 34295 Montpellier Cedex 5 , France. zine@montp.inserm.fr
Cette situation est différente dans l'oreille interne des vertébrés inférieurs et des oiseaux. Dans ces espèces, les cellules ciliées détruites par le bruit ou des substances ototoxiques sont remplacées par de nouvelles cel$5^{\mathrm{e}}$ mois de la vie fœtale. Par la suite, ce nombre de cellules, ridiculement faible pour une fonction aussi sophistiquée que l'audition, ne fait que décroître au fil du temps en raison des agressions d'origine exogène et endogène (drogues ototoxiques, bruits intenses, accidents ischémiques...) qui les altèrent, ou tout simplement de l'âge. Les surdités neurosensorielles touchent près de 22 millions d'européens, soit $6 \%$ de la population et, dans la majorité des cas, elles résultent de lésions entraînant des pertes de $(\rightarrow) \mathrm{m} / \mathrm{s}$ 2004, $n^{\circ} 3$, p. 304 et p. 311 lules ciliées $[1,2]$ qui proviennent en partie d'une réactivation de la prolifération des cellules de soutien. II y a vraisemblablement, dans l'évolution phylogénétique de l'oreille interne des mammifères, un stade où l'épithélium sensoriel perd cette propriété de conserver une population de cellules souches ou celle de permettre leur prolifération et leur différenciation en de nouvelles cellules ciliées. Toutefois, une capacité très limitée de régénération des cellules ciliées subsiste probablement dans le système vestibulaire des mammifères [3]. Depuis 